\title{
High Frequency Limitations of Active Rectifier Circuits for RFID Applications
}

\author{
Julia Fischer, Johan Borg, Jonny Johansson \\ EISLAB, Dept. of Computer Science and Electrical Engineering \\ Luleå University of Technology, SE-971 87 Luleå, Sweden
}

\begin{abstract}
This paper analyses the frequency limitations of an active rectifier for RFID applications that has been optimised for $13.56 \mathrm{MHz}$. The rectifier utilises an active MOS diode with threshold cancellation and a control scheme to reduce reverse leakage. The rectifier is implemented in AMS $0.35 \mu \mathrm{m}$ CMOS and simulated in Cadence Spectre. For an input voltage of $2 \mathrm{~V}$ and an output current of $20 \mu \mathrm{A}$, a power and voltage conversion efficiency of $83 \%$ and $89 \%$, respectively, are achieved at 13.56 MHz. We show that reducing the width of the main MOS transistor from 90 to $60 \mu \mathrm{m}$ improves the upper frequency limit, but beyond $30 \mathrm{MHz}$ the finite speed of the threshold cancellation control circuit limits the efficiency of the rectifier circuit.
\end{abstract}

Index Terms-RFID, rectifier, frequency response, threshold cancellation, reverse leakage, CMOS

\section{INTRODUCTION}

Radio frequency identification (RFID) has been expanding continuously to new areas and applications. Access control, public transport, industrial automation and tracking of materials and goods in production flow [1] [2] are just a few examples. An RFID system consists mainly of two parts: a reader unit and a transponder, also called tag. The latter can either be active or passive. Active tags have their own power supply whereas passive tags are remotely powered by the reader unit through a magnetic or electromagnetic field, depending on the frequency of the RF signal transmitted. The signal is rectified in the tag to provide the necessary DC voltage for the operation of the tag. Since the power level of the transmitted signal is relatively small, it is important to achieve a high voltage and power conversion efficiency (VCE/PCE) of the rectifier in the tag. There are several factors that influence the PCE of rectifiers. Firstly, the fixed turn-on voltage of the diodes does not only define the dead zone where the received induced AC voltage level is not sufficient to power up the tag, it also generates a power loss in the diode which is directly proportional to the conducted current. The turn-on voltage is dependent on the threshold voltage of the diode. Schottky diodes have a very low threshold compared to MOS diodes, but are only available in advanced CMOS processes which increases the costs. One cost-efficient alternative is the use of a threshold cancellation technique [3], [4], [5] to minimise the power loss in the diode and thereby increase the PCE. A more detailed description of a threshold cancellation technique follows in the next section. A second important factor which leads to a decrease in PCE is reverse leakage.
Several control schemes, based on comparators, have also been investigated to minimise this problem [6], [7].

One key challenge in the use of passive tags in industrial harsh environment is the transfer of energy over long distances and to tags of unknown orientation. Previous work on RFID systems for applications in tracking production flows concentrated on the frequency band around $125 \mathrm{kHz}$. It could be beneficial to shift the frequency band to around $13.56 \mathrm{MHz}$ or even higher. Even though the permissible magnetic field power is lower than on $125 \mathrm{kHz}$, but only by $6 \mathrm{~dB}$ (EU directive 2013/752/EU), the frequency is around a factor of 100 higher, which means the induced voltage in an antenna could, theoretically, be up to 50 times higher at $13.56 \mathrm{MHz}$. This in turn means the active diode will be turned on earlier and hence, will lead to lower loss. Another advantage that comes with higher frequencies is the fact that it allows for simpler antennas with lower inductance and thus lower number of turns.

Many publications on rectifier circuits with the aim to improve their operation at $13.56 \mathrm{MHz}$ exist. However, a quantitative comparison is rather difficult as the general conditions like input voltage and output resistance vary a lot. In [6] a full wave active rectifier with $2 \mathrm{~V}$ input and a $500 \Omega$ output resistance achieves a PCE of about $84 \%$. Another active full wave rectifier is presented in [7]. Here a PCE greater than $80 \%$ is reported for input voltages above $2 \mathrm{~V}$. A voltage doubler rectifier with $500 \mathrm{mV}$ input and a $10 \mathrm{k} \Omega$ output resistance is presented in [8]. For this setup the rectifier achieves a PCE of $80 \%$. Some of these works looked briefly into the operation at frequencies above $13.56 \mathrm{MHz}$ but without further investigation of frequency limitations.

In this work a MOS rectifier with threshold cancellation and a reverse leakage control scheme is investigated in terms of its frequency response and limitations for frequencies above $1 \mathrm{MHz}$. The circuit is optimised for $13.56 \mathrm{MHz}$ operation and the limitations incurred as the frequency is increased are analysed. In section II the initial circuit used for the investigation is presented, including a short illustration of the applied threshold cancellation technique and the optimisation of the circuit for $13.56 \mathrm{MHz}$. The frequency response and limitations of the optimised circuit are described in section III. Various steps to increase the frequency range are investigated and the simulation results that were obtained with Cadence Spectre are presented and discussed. Conclusions follow in section IV. 


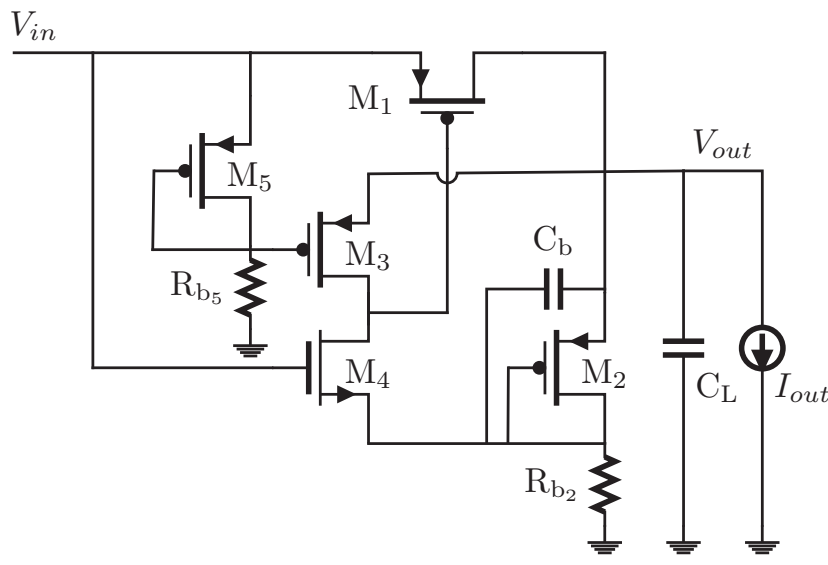

Fig. 1. Initial circuit design for single MOS diode rectifier with threshold cancellation and reverse leakage control [9].

\section{CiRCUit Design}

\section{A. Utilised Rectifier}

As a starting point served the circuit presented in [9], also shown in Fig. 1. It is based on an internal threshold cancellation technique for MOS diodes and, in addition, utilises a control scheme to minimise reverse leakage. The main idea of the threshold cancellation technique is to place a diode connected MOS transistor $\left(\mathrm{M}_{2}\right.$ in Fig. 1) between drain and gate of the MOS diode $M_{1}$ to reduce the effective threshold of it. However, the reverse leakage will increase rapidly when the transistor $\mathrm{M}_{1}$ is reverse-biased if the effective threshold is reduced. To minimise the reverse leakage, a CMOS inverter, $\mathrm{M}_{3}$ and $\mathrm{M}_{4}$, is added. Its purpose is to switch between activating the threshold cancellation during the positive halfperiod of the input signal and turning off $\mathrm{M}_{1}$ completely during the negative half-period. By operating $\mathrm{M}_{1}$ as a reducedthreshold MOS diode rather than as a pure CMOS switch, the exact timing of the turn on and turn off instances becomes less critical. Thus, the need for a fast and highly accurate control circuit is reduced and a simple driver circuit as shown in Fig. 1 has been shown to be sufficient for operation at low frequencies up to $1 \mathrm{MHz}$.

In the original design, which was optimised for $1 \mathrm{MHz}$, the widths of the transistors $\mathrm{M}_{1}, \mathrm{M}_{2}, \mathrm{M}_{3}, \mathrm{M}_{4}$ and $\mathrm{M}_{5}$ were chosen as follows: $200 \mu \mathrm{m}, 1 \mu \mathrm{m}, 5 \mu \mathrm{m}, 5 \mu \mathrm{m}$ and $1 \mu \mathrm{m}$, respectively. The resistor sizes $R_{b 2}$ and $R_{b 5}$ were set to $700 \mathrm{k} \Omega$ and $1 \mathrm{M} \Omega$, respectively.

\section{B. Optimisation for $13.56 \mathrm{MHz}$}

This and all following circuits were implemented in the AMS $0.35 \mu \mathrm{m}$ CMOS process and simulated with Cadence Spectre.

At an operating frequency of $1 \mathrm{MHz}$, the circuit with its original parameters, an input voltage of $2 \mathrm{~V}$ and an output current of $20 \mu \mathrm{A}$ achieved a PCE and VCE of $84.9 \%$ and $92.4 \%$, respectively. The distribution of power dissipation in selected components are shown in table I. The biggest loss contributor is $\mathrm{M}_{1}$ with $42 \%$ of the overall loss. With the frequency increased to $13.56 \mathrm{MHz}$ the PCE and VCE dropped down to $79.2 \%$ and $86.6 \%$, respectively and the power dissipation in $\mathrm{M}_{1}$ increased to $57 \%$ of the overall loss. Fig. 2 shows the voltage signal at the gate of $\mathrm{M}_{1}$ as well as the current through the transistor for both operation at 1 and $13.56 \mathrm{MHz}$. In the latter case it becomes apparent that $\mathrm{M}_{1}$ doesn't switch fast enough, so neither threshold cancellation nor leakage control are working to their full potential as the frequency increases. There are two possibilities to overcome this problem and to thereby increase efficiency; decrease the width of $\mathrm{M}_{1}$ and increase the current that can be sunk by $\mathrm{M}_{4}$ when turning on $\mathrm{M}_{1}$. To achieve the latter, $R_{b 2}$ has to be decreased and $\mathrm{M}_{2}$ scaled accordingly to keep the desired threshold cancellation voltage. This leads to a trade-off because it in turn means a higher current through the resistor and thus higher loss in power. A parametric analysis of the width of $\mathrm{M}_{1}$ shows that decreasing the width to $90 \mu \mathrm{m}$ results in the highest PCE at $13.56 \mathrm{MHz}$. Further parametric analyses were performed to determine the the optimum values for $R_{b 2}$ $(700 \mathrm{k} \Omega)$ and the widths $(3 \mu \mathrm{m})$ of the transistors $\left(\mathrm{M}_{3}\right.$ and $\mathrm{M}_{4}$ ) of the inverter. With the circuit parameters changed to the optimum values, the PCE and VCE could be increased up to $82.9 \%$ and $89.2 \%$, respectively, only slightly below the achieved values at an operating frequency of $1 \mathrm{MHz}$. Still $\mathrm{M}_{1}$ remains the main contributor to the power dissipation, with $50 \%$ of the overall loss.

\section{FREQUENCY LIMITATIONS}

To investigate the optimised circuit's frequency response and its limits, the frequency was first doubled from $13.56 \mathrm{MHz}$ onwards and later some intermediate steps were added. Already at around $30 \mathrm{MHz}$ the PCE and VCE started to drop significantly (Fig. 3). The power loss distribution in Table I shows, that again, $\mathrm{M}_{1}$ is the main contributor with its power loss increased to $62 \%$ of the overall loss at $27.12 \mathrm{MHz}$. Similarly as for the original circuit, the switching speed of $\mathrm{M}_{1}$ is the limiting parameter. Choosing a smaller width increases the switching speed and should in turn result in better performance at higher frequency. But this comes with the cost of slightly lower PCE and VCE when operating at 13.56 MHz, where optimal performance was obtained for a width of $\mathrm{M}_{1}$ of $90 \mu \mathrm{m}$. Choosing a width $W_{1}$ smaller than the optimal width found for $13.56 \mathrm{MHz}$ and no other changes in the circuit's design improved the circuit's behaviour for higher frequencies. For $W_{1}=60 \mu \mathrm{m}$ the loss in PCE at $13.56 \mathrm{MHz}$ compared to the width $W_{1}=90 \mu \mathrm{m}$ is negligibly small $(<0.5 \%)$ but in turn the PCE only starts dropping significantly at around $50 \mathrm{MHz}$ compared to around $30 \mathrm{MHz}$ for the width $W_{1}=90 \mu \mathrm{m}$ as shown in Fig. 4 .

As seen in Table I, with increasing frequency the additional power loss occurs mainly in $\mathrm{M}_{1}$ due to the increase in reverse leakage caused by a delay in the switching time. To further investigate the source of the delayed switching and to investigate the possible upper limits of this circuit configuration, an ideal pulsed voltage source was placed at 
TABLE I

DISTRIBUTION OF POWER DISSIPATION (IN $\mu \mathrm{W}$ AND PERCENTAGE OF OVERALL LOSS) IN SELECTED COMPONENTS AND POWER CONVERSION EFFICIENCY FOR DIFFERENT SETUPS AND FREQUENCIES WITH AVERAGE INPUT POWER OF $44 \mu \mathrm{W}$

\begin{tabular}{|l|r|r|r|r|r|r|r|r|r|}
\hline & \multicolumn{2}{|c|}{ original circuit } & \multicolumn{2}{|c|}{ optimised circuit } & \multicolumn{3}{|c|}{ pulsed V-source @ M1 } & \multicolumn{1}{c|}{ pulsed V-source @ M3/4 } \\
\hline & $1 \mathrm{MHz}$ & $13.56 \mathrm{MHz}$ & $13.56 \mathrm{MHz}$ & $27.12 \mathrm{MHz}$ & $13.56 \mathrm{MHz}$ & $27.12 \mathrm{MHz}$ & $216.96 \mathrm{MHz}$ & $13.56 \mathrm{MHz}$ & $27.12 \mathrm{MHz}$ \\
\hline PCE & $84.9 \%$ & $79.2 \%$ & $82.9 \%$ & $77.0 \%$ & $83.7 \%$ & $83.3 \%$ & $78.3 \%$ & $83.4 \%$ & $77.9 \%$ \\
\hline M1 & $2.5(42 \%)$ & $5.1(57 \%)$ & $3.6(50 \%)$ & $5.9(62 \%)$ & $3.0(47 \%)$ & $3.0(47 \%)$ & $3.1(37 \%)$ & $3.1(47 \%)$ & $5.4(60 \%)$ \\
\hline M3 & $0.5(7 \%)$ & $0.6(7 \%)$ & $0.5(7 \%)$ & $0.6(7 \%)$ & $0.4(6 \%)$ & $0.4(6 \%)$ & $0.5(6 \%)$ & $0.4(7 \%)$ & $0.5(6 \%)$ \\
\hline M4 & $0.3(5 \%)$ & $0.6(7 \%)$ & $0.5(7 \%)$ & $0.6(7 \%)$ & $0.2(3 \%)$ & $0.2(4 \%)$ & $0.5(6 \%)$ & $0.4(6 \%)$ & $0.6(6 \%)$ \\
\hline M5 & $0.1(2 \%)$ & $<0.1(<1 \%)$ & $0.1(1 \%)$ & $<0.1(<1 \%)$ & $0.1(1 \%)$ & $<0.1(<1 \%)$ & $1.5(18 \%)$ & $0.1(2 \%)$ & $0.2(2 \%)$ \\
\hline Rb2 & $1.7(27 \%)$ & $1.9(21 \%)$ & $1.6(22 \%)$ & $1.7(18 \%)$ & $1.5(24 \%)$ & $1.5(24 \%)$ & $2.2(26 \%)$ & $1.6(24 \%)$ & $1.7(19 \%)$ \\
\hline
\end{tabular}

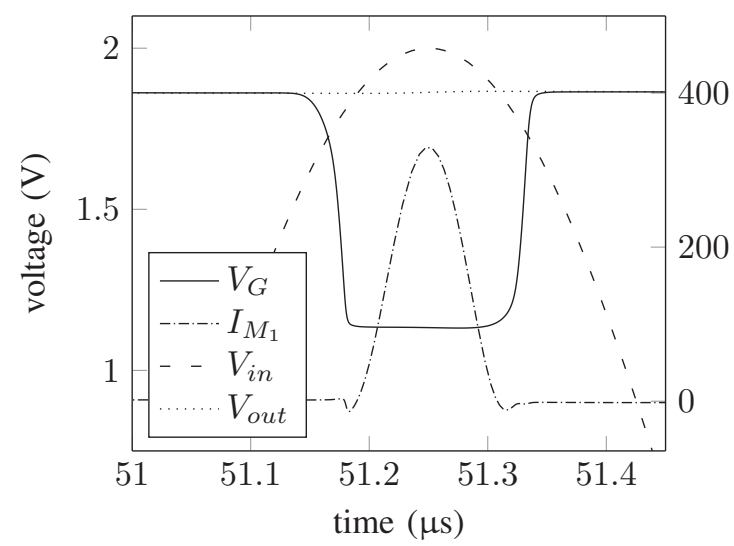

(a)

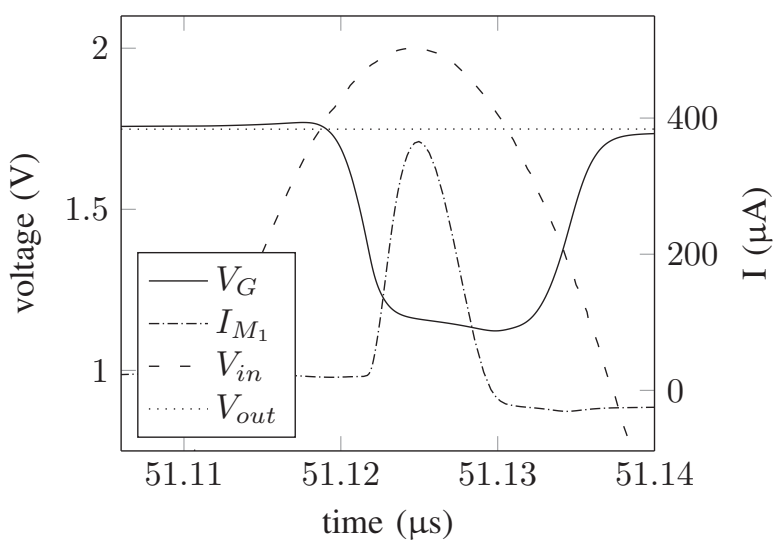

(b)

Fig. 2. Current and gate voltage of $\mathrm{M}_{1}$ plotted together with input and output signal of the original circuit for a) $1 \mathrm{MHz}$ and b) $13.56 \mathrm{MHz}$.

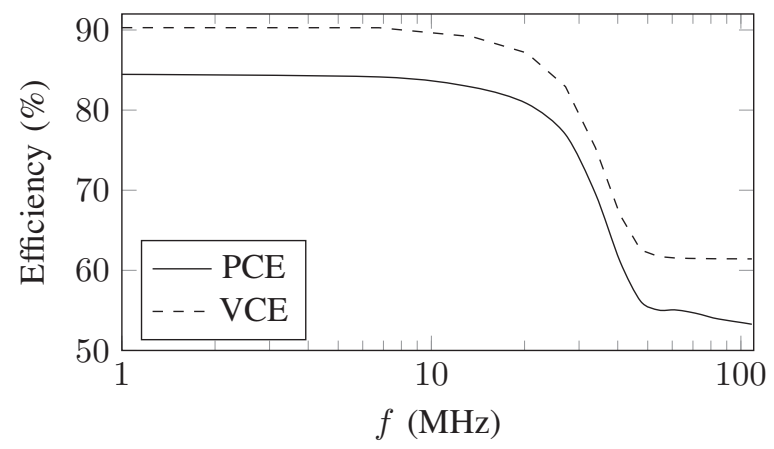

Fig. 3. PCE and VCE versus frequency for transistor width $W_{1}=90 \mu \mathrm{m}$ with $V_{\text {in }}=2 \mathrm{~V}$ and $I_{\text {out }}=20 \mu \mathrm{A}$.

the gate of $\mathrm{M}_{1}$ to ensure precisely timed switching (Fig. 5). The inverter transistors $\mathrm{M}_{3}$ and $\mathrm{M}_{4}$ were kept to include their power dissipation in the simulations for better comparison with the simulations from the optimised circuit. With the ideal pulsed voltage source at gate $\mathrm{M}_{1}$ the PCE could be kept above $80 \%$ for frequencies up to $100 \mathrm{MHz}$. Even then, it only slowly decreased and was above $70 \%$ for frequencies up to about $600 \mathrm{MHz}$. The frequency behaviour is plotted in Fig. 6. The power dissipation in $\mathrm{M}_{1}$ only slowly increased with increasing frequency (about $10 \%$ from 13 to $400 \mathrm{MHz}$ ), whereas a significant increase in power dissipation could be registered for $\mathrm{M}_{5}$, which accounts for the main part of the

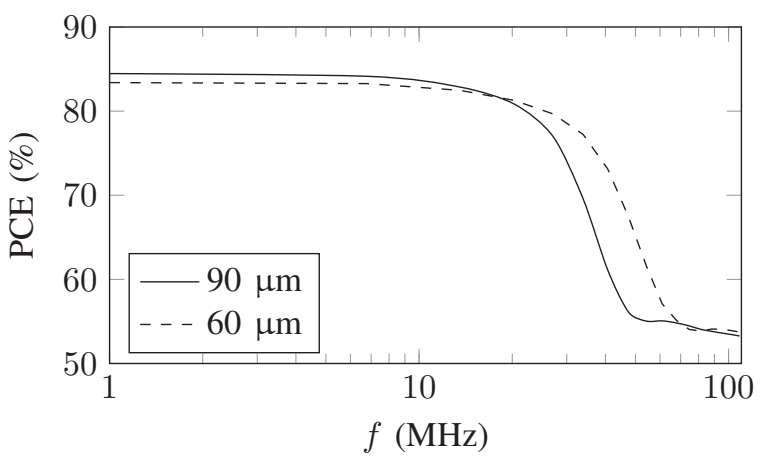

Fig. 4. Comparison of PCE for different widths of transistor $\mathrm{M}_{1}$ : optimum width $(90 \mu \mathrm{m})$ for $13.56 \mathrm{MHz}$ and a smaller width $(60 \mu \mathrm{m})$ for improved frequency response for higher frequencies with $V_{i n}=2 \mathrm{~V}$ and $I_{\text {out }}=20 \mu \mathrm{A}$.

additional loss for higher frequencies in this setup, as shown in Table I. Sharp signal slopes could be established with the pulsed voltage source at the gate of $\mathrm{M}_{1}$. Both the threshold cancellation and the control scheme for minimising the reverse leakage could then work in a proper manner, resulting in high PCE and VCE and showing that the active MOS diode is well capable of operating at high frequencies.

In the next step the ideal pulsed voltage source was moved from $\mathrm{M}_{1}$ to the inverter stage $\mathrm{M}_{3} / \mathrm{M}_{4}$. One pulsed voltage source was placed at the gate of $\mathrm{M}_{3}$ to replace the driver circuit formed by $\mathrm{M}_{5}$ and $\mathrm{R}_{\mathrm{b}_{5}}$. Again, both these components 


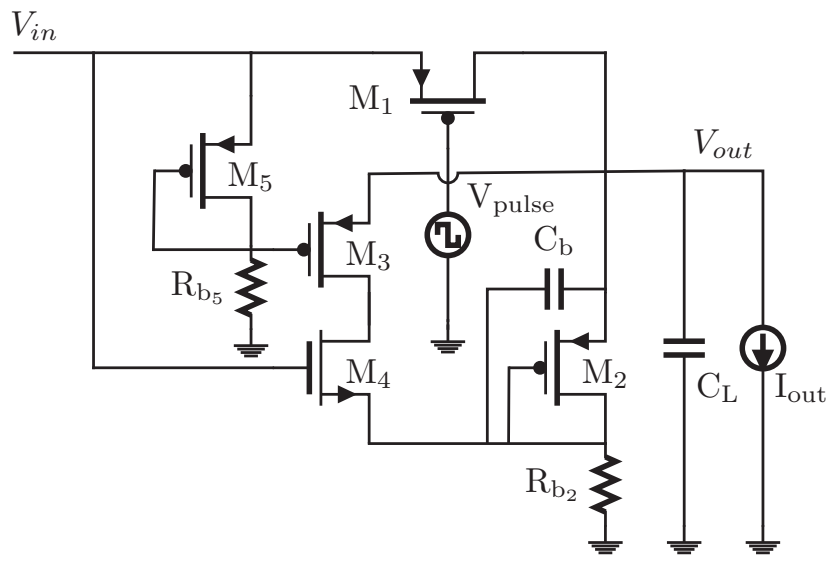

Fig. 5. Modified circuit design with pulsed voltage source at gate of $M_{1}$.

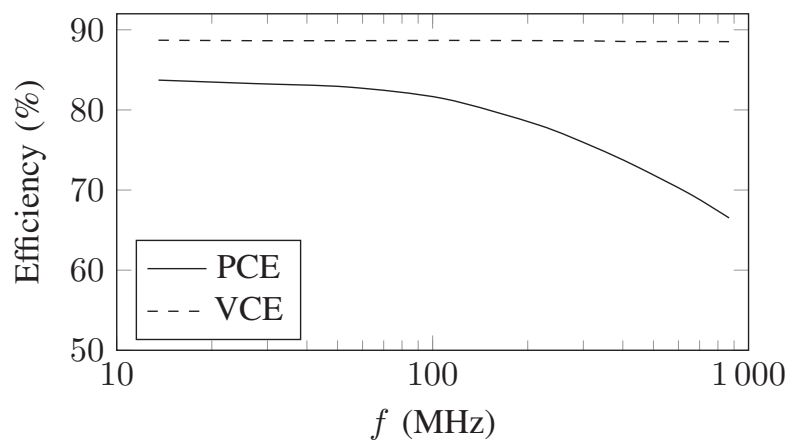

Fig. 6. PCE and VCE versus frequency for modified circuit with pulsed voltage source at gate of $\mathrm{M}_{1}$ with $V_{\text {in }}=2 \mathrm{~V}$ and $I_{\text {out }}=20 \mu \mathrm{A}$.

were kept to include their power dissipation in the simulation for better comparison with the former simulations. Another pulsed voltage source was placed at the gate of $\mathrm{M}_{4}$. With this setup the PCE droped below $80 \%$ at around $25 \mathrm{MHz}$ and below $70 \%$ at around $40 \mathrm{MHz}$. Again, the main part of the loss occurred in $\mathrm{M}_{1}$ due to $\mathrm{M}_{3} / \mathrm{M}_{4}$ not being able to drive the gate of $\mathrm{M}_{1}$ sharply enough. Changing the widths of the inverter did not result in any improvement.

As another attempt to increase the PCE by improving the switching characteristics, two more inverter stages were added in series between the $M_{3} / M_{4}$ stage at the gate of $M_{1}$. The inverters were scaled with the factor $e$. A slight decrease in power loss in $\mathrm{M}_{1}$ due to reduced leakage could be observed, but not enough to make up for the increased power dissipation due to the additional transistors of the additional inverter stages.

\section{CONCLUSION}

The frequency response of an active RFID rectifier was investigated. The circuit used here utilises an active MOS diode with a threshold cancellation technique and a control scheme to minimise reverse leakage. After optimisation and slight modification, the simulations, done with Cadence Spectre in AMS $0.35 \mu \mathrm{m}$ CMOS, showed that the rectifier is well suitable for RFID applications operating at $13.56 \mathrm{MHz}$ as it reaches both high PCE $(83 \%)$ and VCE $(89 \%)$. For frequencies up to $30 \mathrm{MHz}$ it still shows acceptable performance with a PCE well above $70 \%$. It was also shown that the active MOS diode is well capable of operating at high frequencies if sharp switching characteristics can be established at its gate. The key challenge for this lies in the steering of the signal which is generated by the preceding inverter stage.

\section{ACKNOWLEDGEMENT}

This work has received funding from the European Union's Horizon 2020 Research and Innovation Programme under the Grant Agreement No. 636834, DISIRE.

\section{REFERENCES}

[1] B. Kvarnström, B. Bergquist, and K. Vännman. "RFID to improve traceability in continuous granular flows: An experimental case study". In: Quality Engineering 23.4 (2011), pp. 343-357.

[2] J. Rabe, P. Fouche, and K. O'Neill. "Development of a RF tracer for use in the mining and minerals processing industry". In: The Third Southern African Conference on Base Metals. 2005.

[3] H. Raben, J. Borg, and J. Johansson. "Improved efficiency in the CMOS cross-connected bridge rectifier for RFID applications". In: Mixed Design of Integrated Circuits and Systems (MIXDES), 2011 Proceedings of the 18th International Conference. June 2011, pp. 334-339.

[4] K. Kotani and T. Ito. "High efficiency CMOS rectifier circuits for UHF RFIDs using Vth cancellation techniques". In: ASIC, 2009. ASICON '09. IEEE 8th International Conference on. Oct. 2009, pp. 549-552.

[5] P.T. Theilmann et al. "Near zero turn-on voltage high-efficiency UHF RFID rectifier in silicon-on-sapphire CMOS". In: Radio Frequency Integrated Circuits Symposium (RFIC), 2010 IEEE. May 2010, pp. 105108.

[6] Y. Lu and W.-H. Ki. "A 13.56 MHz CMOS Active Rectifier With Switched-Offset and Compensated Biasing for Biomedical Wireless Power Transfer Systems". In: Biomedical Circuits and Systems, IEEE Transactions on 8.3 (June 2014), pp. 334-344.

[7] H.-M. Lee and M. Ghovanloo. "An Integrated Power-Efficient Active Rectifier With Offset-Controlled High Speed Comparators for Inductively Powered Applications". In: Circuits and Systems I: Regular Papers, IEEE Transactions on 58.8 (Aug. 2011), pp. 1749-1760.

[8] D. Nguyen et al. "A high power conversion efficiency rectifier with new internal VTh cancellation topology for RFID applications". In: Communications and Electronics (ICCE), 2014 IEEE Fifth International Conference on. July 2014, pp. 313-316.

[9] H. Raben, J. Borg, and J. Johansson. "An active MOS diode with Vth-cancellation for RFID rectifiers". In: RFID (RFID), 2012 IEEE International Conference on. Apr. 2012, pp. 54-57. 